\title{
ORDER EXTENSION OF ORDER MONOMORPHISMS ON A PREORDERED TOPOLOGICAL SPACE
}

\section{GHANSHYAM MEHTA}

Department of Economics

The University of Queensland

Qld. 4072, Australia

(Received April 29, 1992)

ABSTRACT. This paper proves Nachbin-type extension theorems for infinitely many functions on a topological space equipped with a preorder.

KEYWORDS AND PHRASES. Order extension, order monomorphism. 1991 AMS SUBJECT CLASSIFICATION CODES. 54F05, 90 A10.

\section{INTRODUCTION.}

The well-known extension theorem of Nachbin [1, p. 36] gives conditions under which a realvalued continuous order-homomorphism defined on a closed subset of a normally preordered space $E$ can be extended to a real-valued continuous order homomorphism on the whole space. In the particular case in which the preorder on the space is the discrete order, the Nachbin extension theorem reduces to the Urysohn-Tietze extension theorem on normal spaces.

The objective of this paper is to study the following variant of the extension problem considered by Nachbin. Suppose that instead of a single real-valued continuous order homomorphism, one has a collection $F=\left\{f_{i}\right\}_{i \in I}$ of real-valued continuous order monomorphisms, where $f_{i}$ is defined on a closed subset $D_{i}$ of a preordered topological space $E$. Then the problem is to find a real-valued continuous order-monomorphism $f$ on the whole space $E$ that is an order-extension of each order monomorphism $f_{i}$ in the collection $F$. In this paper, we give sufficient conditions for the existence of such a 'universal' order-extension.

A special case of the problem studied in this paper arises in mathematical economics in the context of the Euclidean distance approach used by Arrow and Hahn [2] to construct a continuous order monomorphism on a convex subset $X$ of $R^{n}$ equipped with a total preorder. The Arrow-Hahn method [2, pp. 82-87] consists of taking a point $x_{0}$ and then by defining the 'utility' of $x$ to be the Euclidean distance from $x_{0}$ to the upper section of $x$. This Arrow-Hahn function can be shown to be continuous under certain conditions. In this way, one is given a collection of continuous order monomorphisms $\left\{f_{x}: x \in D\right\}$ defined on the upper sections of the points $x$ in $D$. Now the problem is to 'construct' a continuous order monomorphism $f$ on the whole space $X$. It should be observed that the problem is not merely to show the existence of a continuous order monomorphism since, in the finite-dimensional Arrow-Hahn context, this can be deduced concisely from general topological theorems such as Eilenberg's theorem ([3] or Corollary 1 of [4]). The problem is to somehow 'construct' the function $f$ as an order-extension of the functions $f_{x}$ for $x$ in $D$. See Mehta $[5,6]$ for further discussion of the Arrow-Hahn theorem.

For some recent work on the separation and extension theorems of Nachbin in preordered topological spaces the reader is referred to Herden [7,8]. 


\section{PRELIMINARIES.}

Let $E$ be a set. A preorder $\leq$ on $E$ is a reflexive and transitive binary relation on $E$. A preordered set is a set equipped with a preorder. A preorder $\leq$ on $E$ gives rise to two other binary relations on $E$ as follows. If $x, y \in E$ then $x \sim y$ if and only if $x \leq y$ and $y \leq x$. The relation $\sim$ is an equivalence relation. If $x, y \in E$ then $x<y$ if and only if $x \leq y$ and not $y \leq x$. The relation $<$ is irreflexive and transitive.

A preorder $\leq$ is said to be an order if it is antisymmetric. A preorder $\leq$ is said to be total if for every two elements $x, y \in E$ either $x \leq y$ or $y \leq x$.

A subset $X$ of a preordered set $E$ is said to be decreasing if $b \in X, a \leq b$ imply that $a \in X$. Each subset $X$ of $E$ determines, in a unique manner, a decreasing set $d(X)$ which is the smallest one among the decreasing sets containing $X$. Dually, one defines the concepts of an increasing set and the smallest increasing set $i(X)$ containing a given subset $X$.

Let $E$ be a totally preordered set. Consider the following collection of subsets of $E$ :

(1) $(a, b)=\{x \in E: a<x<b\}$;

(2) $\left(a, b_{0}\right]=\{x \in E: a<x \leq b\}$ if $b_{0}$ is the last element of $E$;

(3) $\left[a_{0}, b\right)=\left\{x \in E: a_{0} \leq x<b\right\}$ if $a_{0}$ is the first element of $E$.

Then this collection of subsets of $E$ is a basis for a topology on $E$ called $\leq$-order topology.

Let $E$ be a topological space equipped with a preorder $\leq$. Each subset $X$ of $E$ determines uniquely a closed decreasing set $D(X)$ which is the smallest among the closed decreasing sets containing $X$. Analogously, we define the concept of the smallest closed increasing set $I(X)$ containing a given subset $X$. If $A$ and $B$ are two subsets of $E$ we write $A<B$ to indicate that $D(A) \cap I(B)=\phi$.

Let $E$ be a topological space equipped with a preorder $\leq$. The preorder $\leq$ is said to be continuous if $d(y)=\{x \in E: x \leq y\}$, the lower section of $y$, and $i(y)=\{x \in E: y \leq x\}$, the upper section of $y$, are closed in $E$ for every $y \in E$. The preorder is said to be strongly continuous if $d(X)$ and $i(X)$ are closed in $E$ for every closed subset $X$ of $E$.

A topological space $E$ equipped with a preorder $\leq$ is said to be normally preordered if for every two disjoint closed subsets $F_{0}$ and $F_{1}$ of $E, F_{0}$ being decreasing and $F_{1}$ increasing, there exist two disjoint open subsets $A_{0}$ and $A_{1}$ such that $A_{0}$ contains $F_{0}$ and is decresing and $A_{1}$ contains $F_{1}$ and is increasing.

Let $E_{1}$ and $E_{2}$ be two preordered sets. A function $f$ on $E_{1}$ to $E_{2}$ is said to be an order homomorphism (or isotone) if $x, y \in E$ and $x \leq y$ imply that $f(x) \leq f(y)$. A function $f$ on $E_{1}$ to $E_{2}$ is said to be an order monomorphism if it is an order homomorphism and $x<y$ implies that $f(x)<f(y)$.

Let $E, H$ be two preordered sets and $F$ a subset of $E$. Let $g: F \rightarrow H$ be an order monomorphism. Then an order monomorphism $f: E \rightarrow H$ is said to be an order extension of $g$ if it satisfies the following conditions:

(i) $g(a) \leq g(b)$ and $a, b \in F$ imply that $f(a) \leq f(b)$

(ii) $g(a)<g(b)$ and $a, b \in F$ imply that $f(a)<f(b)$.

3. ORDER EXTENSION OF ORDER MONOMORPHISMS.

The following fundamental theorem on the extension of an order homomorphism is due to Nachbin [1, p. 36].

(Nachbin's Extension Theorem) Let $E$ be a normally preordered space $F \subseteq E$ a closed subset and $f$ a bounded real-valued function which is continuous and isotone on $F$. We shall indicate by $A(r)$ the set of points $x$ in $F$ such that $f(x) \leqq r$ and $B(r)$ the set of points $x$ in $F$ such that $f(x) \geqq r$, 
where $r$ is a real number. Then in order that the function $f$ may be extended to $E$ in such a way as to become a bounded, continuous and isotone real function on $E$ it is necessary and sufficient that $r<r^{\prime}$ implies $A(r)<B\left(r^{\prime}\right)$.

We now use Nachbin's extension theorem to prove the following theorem on extensions of order monomorphisms.

THEOREM 1. Let $E$ be a $T_{1}$ topological space equipped with a preorder $\leq$ such that the following conditions are satisfied:

(i) $\boldsymbol{E}$ is normally preordered;

(ii) The preorder $\leq$ is strongly continuous;

(iii) For each $x \in E$, the set $\{a \in E: a<x\}$ is open in $E$;

(iv) $F=\left\{D_{n}\right\}_{n=1}^{\infty}$ is a countable family of closed increasing subsets of $E$ such that for each $n=1,2, \cdots$ there exists a real-valued continuous order-monomorphism $f_{n}$ defined on $D_{n}$;

(v) There exists a countable and topologically dense subset $Z=\left\{z_{n}\right\}_{n=1}^{\infty}$ of $E$ with the property that $z_{n} \in D_{n}$ for all $n$.

Then there exists a real-valued continuous order monomorphism $f$ on $E$ that is an order extension of $f_{n}$ for each positive integer $n$.

PROOF. By hypothesis, for each $n=1,2, \cdots$ there exists a real-valued continuous order monomorphism $f_{n}$ on $D_{n}$. Since the extended real line $\bar{R}$ is order homeomorphic to $[0,1]$, we may assume without loss of generality that for each positive integer $n, f_{n}: D_{n} \rightarrow[0,1]$.

We prove next that for each $n=1,2, \cdots$ the conditions of Nachbin's extension theorem [9, p.36] are satisfied for the function $f_{n}$. To this end, let $r, r^{\prime}$ be two real numbers such that $r<r^{\prime}$. Define $A_{n}(r)=\left\{x \in D_{n}: f_{n}(x) \leqq r\right\} \quad$ and $B_{n}\left(r^{\prime}\right)=\left\{x \in D_{n}: f_{n}(x) \geqq r^{\prime}\right\}$. We claim that $D\left(A_{n}(r)\right) \cap I\left(B_{n}\left(r^{\prime}\right)\right)=\phi$ for every positive integer $n$.

We prove first that $d\left(A_{n}(r)\right) \cap i\left(B_{n}(r)\right)=\phi$ for every positive integer $n$. Let $m$ be a fixed positive integer. If either $A_{m}(r)$ or $B_{m}\left(r^{\prime}\right)$ is empty the result holds. So we may assume that $A_{m}(r)$ and $B_{m}\left(r^{\prime}\right)$ are nonempty. Now suppose that $d\left(A_{m}(r)\right) \cap i\left(B_{m}\left(r^{\prime}\right)\right) \neq \phi$. Then $z \in d\left(A_{m}(r)\right) \cap i\left(B_{m}\left(r^{\prime}\right)\right)$ for some $z \in E$. Since $z \in d\left(A_{m}(r)\right)$ there exists $a \in A_{m}(r)$ such that $z \leq a$. Similarly, there exists $b \in B_{m}\left(r^{\prime}\right)$ such that $b \leq z$. Since $a \in A_{m}(r)$ and $b \in B_{m}\left(r^{\prime}\right)$ we have $f_{m}(a) \leqq r<r^{\prime} \leqq f_{m}(b)$. On the other hand, $b \leq z$ and $z \leq a$ imply that $b \leq a$ by transitivity of the preorder. Therefore, $f_{m}(b) \leqq f_{m}(a)$ because $f_{m}$ is an order homomorphism. This contradiction proves that for every positive $n, d\left(A_{n}(r)\right) \cap i\left(B_{n}\left(r^{\prime}\right)\right)=\phi$.

Now the preorder $\leq$ is strongly continuous. This implies that $d\left(A_{n}(r)\right)$ is a closed decreasing set in $E$ and $i\left(B_{n}\left(r^{\prime}\right)\right)$ is a closed increasing set in $E$ for every positive integer $n$. Therefore, $D\left(A_{n}(r)\right) \subset d\left(A_{n}(r)\right)$ and $I\left(B_{n}\left(r^{\prime}\right)\right) \subset i\left(B_{n}\left(r^{\prime}\right)\right)$. Hence, $D\left(A_{n}(r)\right) \cap I\left(B_{n}\left(r^{\prime}\right)\right)=\phi$ for all $n$.

Thus all the conditions of Nachbin's extension theorem $[1$, p.36] are satisfied and we may conclude that there exists a real-valued continuous order homomorphic extension $g_{n}$ of $f_{n}$ to $E$. Clearly, we may assume that $g_{n}: E \rightarrow\left[0,2^{-n}\right]$.

Define $f: E \rightarrow R$ by $f(x)=\sum_{n=1}^{\infty} g_{n}(x)$ for $x \in E$. Clearly, $f$ is a real-valued continuous order homomorphism on $E$.

To prove that $f$ is an order monomorphism let $x, y$ be two elements in $E$ with $x<y$. Suppose first that $E$ has no least element. This implies that the set $\{a \in E: a<x\}$ is nonempty. It is open by condition (iii). Since $Z$ is dense in $E$ there exists a positive integer $n$ such that $z_{n}$ belongs to $\{a \in E: a<x\}$. Furthermore since $g_{n}$ is an order monomorphism on the increasing set $D_{n}$ and $z_{n} \in D_{n}$, we have $g_{n}(x)<g_{n}(y)$. Hence, $f(x)<f(y)$.

Suppose now that $E$ has a least element $e_{0}$. We may assume that there is no positive integer 
$n$ such that $e_{0} \sim z_{n}$ because in that case there is nothing to be proved. If $e_{0}<x$ the set $\{a \in E: a<x\}$ is nonempty and open. Then arguing as before we have $f(x)<f(y)$. If $x \sim e_{0}$ the set $\{a \in E: a<y\}$ is nonempty and open. Therefore, it contains an element $z_{n}$ of $z$. Again, without loss of generality, we may assume that $e_{0}<z_{n}<y$. Since $g_{n}$ is an order homomorphism on $E$ we have $g_{n}\left(e_{0}\right) \leqq g_{n}\left(z_{n}\right)$. Now because $g_{n}$ is an order monomorphism on the increasing set $D_{n}$ we have $g_{n}\left(z_{n}\right)<g_{n}(y)$. Hence, $g_{n}(x)=g_{n}\left(e_{0}\right) \leqq g_{n}\left(z_{n}\right)<g_{n}(y)$. This implies that $f(x)<f(y)$.

We have proved that for all $x, y$ in $E$ such that $x<y$ we have $f(x)<f(y)$. Consequently, $f$ is a continuous order monomorphism on $E$. Finally, it is clear that $f$ is an order extension of $f_{n}$ for all $n$. This completes the proof of the theorem.

q.e.d.

REMARK 1. It is worth observing that in a compact ordered space the preorder is necessarily strongly continuous $[1, \mathrm{p} .44]$.

REMARK 2. The strong continuity condition has been used in [9] to prove generalizations of Katetov's theorem on the interpolation of a continuous function between semi-continuous functions. For further applications of this condition the reader is referred to [10,11]. A related condition has been used in the theory of order compactifications $[12,13,14]$.

Another important special case of the preceding theorem occurs when $E$ is a totally preordered set with the order topology. Under these conditions, the preorder is strongly continuous as we now prove.

PROPOSITION 1. Let $E$ be a set, $\leq$ a total preorder on $E$ and assume that $E$ has the $\leq$ order topology. Then the preorder is strongly continuous.

PROOF. Let $K$ be a closed subset of $E$. We claim that $d(K)$ is closed. If $d(K)$ is not closed there exists a net $\left\{x_{k}, k \in D\right\}$ in $d(K)$ that converges to $x \notin d(K)$. This implies that $x \notin K$. Therefore, there exists an open set $V$ such that $x \in V$ and $V \cap K=\phi$ because $K$ is closed. Since $E$ has the order topology we may conclude that there is a basis element $B$ such $x \in B \subset V$. We have to consider the following three cases:

1. $x \in B=(a, b)$

2. $x \in B=\left(a, b_{0}\right]$

3. $x \in B=\left[a_{0}, b\right)$

Observe, in the first two cases, that $a<x_{k_{0}}$ for some $k_{0} \in D$ because the net $\left\{x_{k}, k \in D\right\}$ converges to $x$. Since $x_{k_{0}} \in d(K), x_{k_{0}} \leq v$ for some $v \in K$. Now because the preorder is total either $x \leq v$ or $v<x$. If $x \leq v$ then $x \in d(K)$ because $v \in K$. Since $x \notin d(K)$ by hypothesis, we may conclude that $v<x$. Hence, we have $a<x_{k_{0}}<v<x$. This implies that $v \in V$ which is a contradiction because $V \cap K=\phi$.

It remains to consider the third case. So suppose that $x \in B=\left[a_{0}, b\right)$. If $a_{0}<x<b$ then we are in the case just considered above. So let $x=a_{0}$. Since $B$ is an open set there exists $k_{1}$ such that $x_{k_{1}} \in\left[a_{0}, b\right)$. This implies that $a_{0}<x_{k_{1}}$ because $x_{k_{1}} \in d(K)$ and $x \notin d(K)$. Hence, $a_{0}=x<x_{k_{1}} \leq \nu$ for some $v \in K$ since $x_{k_{1}} \in d(K)$. Therefore, $x \in d(K)$ which is a contradiction.

We have proved that in all cases, $K$ closed in $E$ implies that $d(K)$ is also closed. Similarly, we prove that $i(K)$ is closed. Therefore, the preorder $\leq$ is strongly continuous.

Suppose now that $(E, t)$ is a topological space and $\leq$ is a continuous total preorder on $E$. Since $t$ is, in general, finer than the order topology, the above method of proof does not work. However, a simple direct argument based on the Gluing Lemma of topology may be used instead as we now prove.

THEOREM 2. Let $E$ be a topological space equipped with a total preorder $\leq$ such that the following conditions are satisfied: 
(i) The preorder $\leq$ is continuous;

(ii) $F=\left\{D_{n}\right\}_{n=1}^{\infty}$ is a countable family of closed increasing subsets of $E$ such that for each $n=1,2, \ldots$ there exists a real-valued continuous order-monomorphism $f_{n}$ defined on $D_{n}$;

(iii) There exists a countable and topologically dense subset $Z=\left\{z_{n}\right\}_{n=1}^{\infty}$ of $E$ with the property that $z_{n} \in D_{n}$ for all $n$.

Then there exists a real-valued continuous order monomorphism $f$ on $E$ that is an order extension of $f_{n}$ for each positive integer $n$.

PROOF. Without loss of generality, we may assume that for all $n, f_{n}: D_{n} \rightarrow[0,1]$. Let $m$ be an arbitrary but fixed positive integer. We want to show that there exists a real-valued continuous homomorphism $g_{m}$ on $E$ such that $g_{m}(x)=f_{m}(x)$ for all $x \in D_{m}$.

To this end, we claim that either there exists some point $y_{m} \in E$ such that $i\left(y_{m}\right)=D_{m}$ or $E \backslash D_{m}$ is closed. So suppose that $i(a) \neq D_{m}$ for all $a \in E$. We need to prove that $E \backslash D_{m}$ is closed. If $E \backslash D_{m}$ is not closed there exists a net $\left\{x_{d}: d \in D\right\}$ in $E \backslash D_{m}$ that converges to $x \in D_{m}$. Since $i(x) \neq D_{m}$ there exists $v \in D_{m}$ such that $v<x$ because the preorder $\leq$ is total. The set $\{a \in E: v<a\}$ is increasing, open (because the preorder is continuous) and contains $x$. Therefore, there exists $d_{0}$ such that $d_{0} \leq d$ implies that $x_{d} \in\{a \in E: v<a\} \subset D_{m}$. This contradiction proves that $E \backslash D_{m}$ is closed, and completes the proof of the claim.

To show that there is a continuous order-homomorphism $g_{m}$ on $E$ extending $f$, we have to consider the following cases. Suppose first that $E \backslash D_{m}$ is closed. Define a real-valued function $g_{m}$ on $E$ by

$$
\begin{aligned}
g_{m}(x) & =f_{m}(x) & & \text { if } x \in D_{m} \\
& =0 & & \text { if } x \in E \backslash D_{m}
\end{aligned}
$$

Since both $D_{m}$ and $E \backslash D_{m}$ are closed, the Gluing Lemma [15, pp.14-15] implies that $g_{m}$ is continuous. Clearly, $g_{m}$ is an order-homomorphism on $E$.

Suppose now that for some $y_{m} \in E, D_{m}=i\left(y_{m}\right)$. Clearly, $y_{m} \in D_{m}$. Define a real-valued function $g_{m}$ on $E$ by

$$
\begin{aligned}
g_{m}(x) & =f_{m}(x) & & \text { if } x \in i\left(y_{m}\right) \\
& =f_{m}\left(y_{m}\right) & & \text { if } x \in d\left(y_{m}\right)
\end{aligned}
$$

Observe that for all $x, y \in D_{m}$ such that $x \sim y$ we have $f_{m}(x)=f_{m}(y)$ because $f_{m}$ is an order homomorphism. Therefore, for all $x \in d\left(y_{m}\right) \cap i\left(y_{m}\right)$, we have $f_{m}(x)=f_{m}(y)$. Consequently, the Gluing Lemma again implies that $g_{m}$ is continuous. Clearly, $g_{m}$ is an order homomorphism on $E$.

We have proved that for each $n=1,2, \ldots$ there exists a real-valued continuous order homomorphism $g_{n}$ on $E$ such that $g_{n}(x)=f_{n}(x)$ for all $x \in D_{n}$. We may assume without loss of generality that $g_{n}: E \rightarrow\left[0,2^{-n}\right]$.

Finally, observe that sets of the form $\{a \in E: a<x\}$ are open in $E$ for each $x \in E$ because the preorder is continuous and total. Hence, condition (iii) of Theorem 1 is satisfied. Arguing as in the last part of Theorem 1, we may conclude the proof of the theorem.

q.e.d.

REMARK 3. It should be noted that the conditions of Theorem 2 imply that $E$ is normally preordered [4, Proposition 1]. On the other hand, the assumption of strong continuity is not needed because the preorder is total.

\section{REFERENCES}

1. NACHBIN, L., Topology and Order, Van Nostrand, Princeton, N.J., 1965.

2. ARROW, K. \& HAHN, F., General Competitive Analysis, Oliver and Boyd, Edinburgh, 1971.

3. EILENBERG, S., Ordered topological spaces, Amer. J. Math., 63 (1941), 39-45. 
4. MEHTA, G., Existence of an order preserving function on normally preordered spaces, Bull. Austral. Math. Soc. 34 (1986, 141-147.

5. MEHTA, G., A new extension procedure for the Arrow-Hahn theorem, Int. Econom. Rev. 22 (1981), 113-118.

6. MEHTA, G., Utility functions on preordered normed linear spaces, Appl. Math. Lett. 4 (1991), 53-55.

7. HERDEN, G., On the existence of utility functions, Math. Social Sciences 17, (1989), 297-313.

8. HERDEN, G., On a lifting theorem of Nachbin, Math. Social Sciences 19 (1990), 37-44.

9. PRIESTLEY, H., Separation theorems for semi-continuous functions on normally ordered topological spaces, J. London Math. Soc. 3 (1971), 371-377.

10. KUNZI, H., Completely regular ordered spaces, Order $I$ (1990), 283-293.

11. PRIESTLEY, H., Ordered topological spaces and the representation of distributive lattices, Proc. London Math. Soc. 3 (1972), 507-530.

12. KENT, D.C., On the Wallman ordered compactification, Pac. J. Math. 82 (1985), 159-163.

13. KENT, D.C. \& RICHMOND, T., Separation properties of the Wallman ordered compactification, Internat. J. Math. \& Math. Sci. 13 (1990), 209-222.

14. GAMON, M. \& KENT, D.C., A note on ordered planes, Internat. J. Math. \& Math. Sci. 15 (1992), 199-202.

15. ROTMAN, J., An Introduction to Algebraic Topology, Springer-Verlag, New York, 1988. 


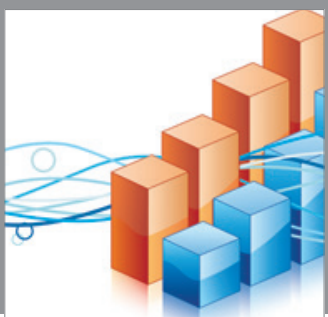

Advances in

Operations Research

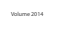

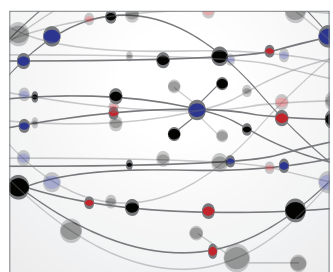

\section{The Scientific} World Journal
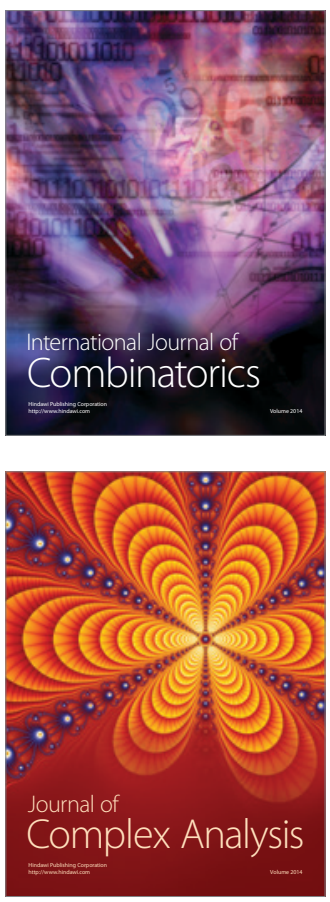

International Journal of

Mathematics and

Mathematical

Sciences
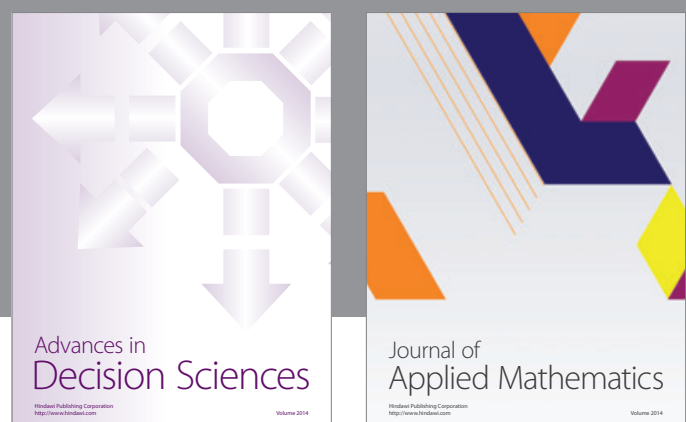

Journal of

Applied Mathematics
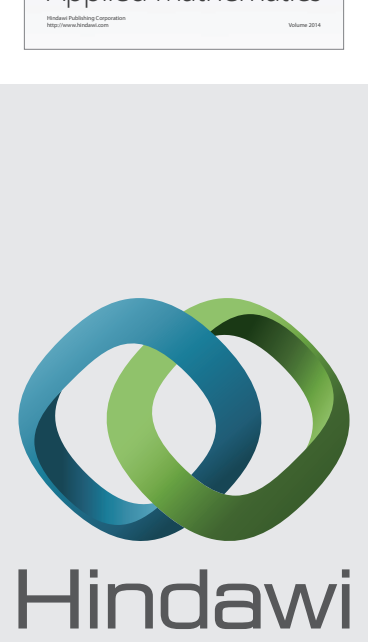

Submit your manuscripts at http://www.hindawi.com
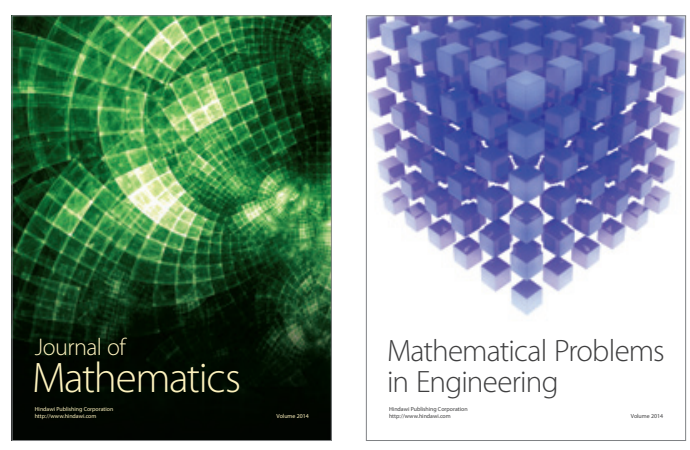

Mathematical Problems in Engineering
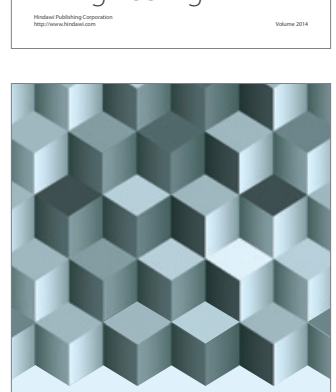

Journal of

Function Spaces
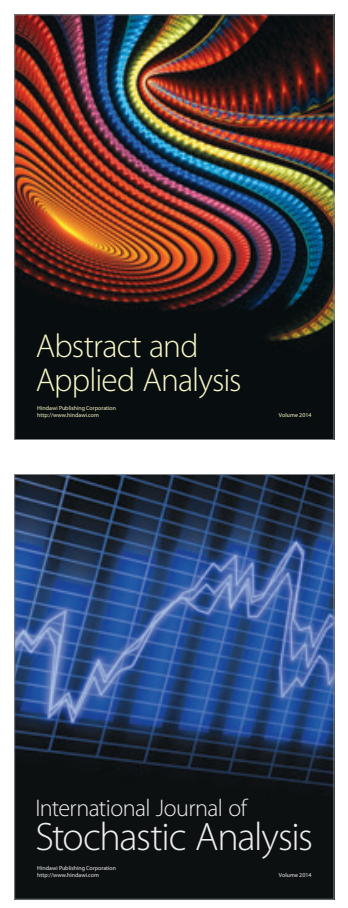

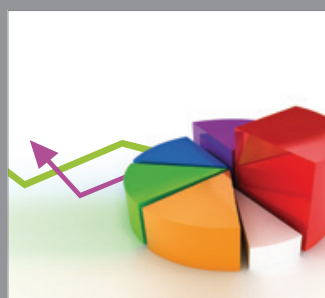

ournal of

Probability and Statistics

Promensencen
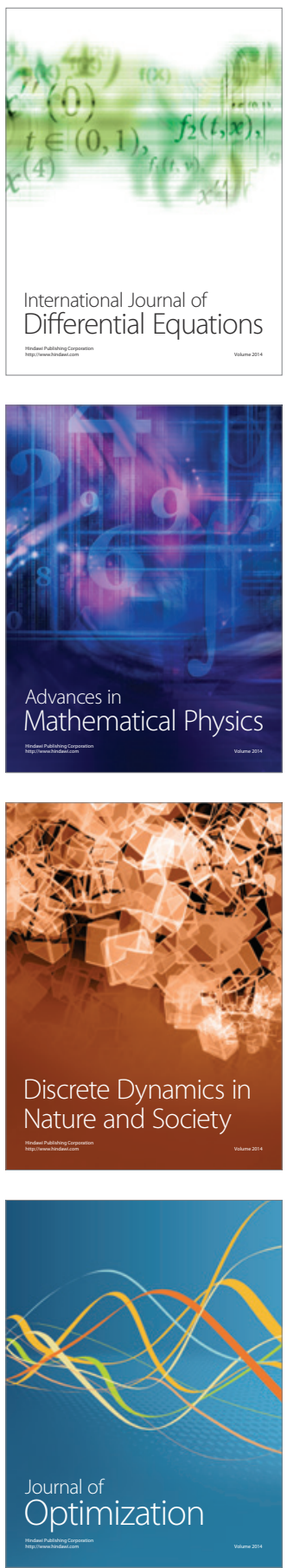